\title{
Modulation of the Inhibitory Substrate Properties of Oligodendrocytes by Platelet-Derived Growth Factor
}

\author{
Dirk M. Lang, ${ }^{1}$ Michael G. Hille, ${ }^{1}$ Martin E. Schwab, ${ }^{2}$ and Claudia A. O. Stuermer ${ }^{1}$ \\ ${ }^{1}$ Faculty of Biology, University of Konstanz, D-78434 Konstanz, Germany, and 2/nstitute for Brain Research, University of \\ Zürich, CH-8029 Zürich, Switzerland
}

Although growth cones typically collapse after encountering 01/galactocerebroside (GalC)-positive oligodendrocytes, the majority of growth cones traversed oligodendrocytes, which were raised for $8-10 \mathrm{~d}$ in medium containing $10 \mathrm{ng} / \mathrm{ml}$ plateletderived growth factor (PDGF). Oligodendrocytes raised 8-10 d in control medium caused growth cone collapse as they normally do, but failed to elicit this response after being transferred to PDGF-containing medium for an additional 8-10 d. The opposite was observed when PDGF-treated oligodendrocytes were brought to control medium. Growth cones collapsed when contacting these cells. Oligodendrocytes also lost their collapse-inducing activity when raised in medium conditioned by astrocytes, known to produce PDGF. Antibody IN-1 is directed against neurite growth inhibitors $(\mathrm{NI})$, proteins of 35 and $250 \mathrm{kDa}$ on the surface of O1/GalC-positive oligodendrocytes, which are known to elicit growth cone collapse. IN-1 immunoreactivity was markedly reduced in PDGF-treated oligodendrocytes. However, both PDGF-treated and control oligodendrocytes exhibited myelin-associated glycoprotein, proteolipid protein, and myelin basic protein immunoreactivity. This suggests that PDGF-treatment affects $\mathrm{NI}$ expression but does not interfere with the expression of advanced myelin marker proteins. Because $\mathrm{NI}$ cause growth cone collapse, the loss of collapse-inducing activity by PDGF-treated oligodendrocytes suggests that PDGF regulates, directly or indirectly, the expression of these proteins.

Key words: oligodendrocyte; loss of inhibitory properties; PDGF-treatment; growth cone collapse; IN-1 immunoreactivity; astrocyte-conditioned medium
Neurite growth inhibitors (NI) elicit collapse of growth cones and retraction of axons in vitro and impair the regrowth of injured CNS fiber tracts in vivo (Schwab et al., 1993). These inhibitors are found on the surface of oligodendrocytes and CNS myelin and are proteins of 35 and $250 \mathrm{kDa}$ (Caroni and Schwab, 1988a; Schwab and Caroni, 1988). Strategies for enhancing axonal regeneration, therefore, involve overcoming the inhibitors' action or, if possible, hindering their expression.

The first approach has been successful: the action of the inhibitors can be partially neutralized in vitro (Caroni and Schwab, 1988b) and in vivo (Schnell et al., 1994) by applications of the antibody IN-1. Because the myelin-associated NI are not yet cloned, the pathways regulating their expression are still unknown. Also, the onset of expression is not precisely defined. Nevertheless, it has been noted that oligodendrocytes in vitro expressing the differentiation marker galactocerebroside (GalC) and exhibiting a highly branched morphology (Schwab and Caroni, 1988) have acquired inhibitory properties, as judged from growth cone reactions after contact with oligodendrocytes (Fawcett et al., 1989; Bandtlow et al., 1990; Bastmeyer et al., 1991).

During further differentiation, oligodendrocytes express additional proteins, including proteolipid protein (PLP), myelin basic protein (MBP), and myelin-associated glycoprotein (MAG), all of

Received May 7, 1996; revised June 21, 1996; accepted June 28, 1996.

This work was supported by Deutsche Forschungsgemeinschaft Grant Stu112/10 to C.A.O.S. and the Gemeinnützige Hertie-Stiftung. D.M.L. is a fellow of the Boehringer-Ingelheim Fonds. We thank Drs. M. Bastmeyer, C. Erxleben, and J. Ostheim for help with the image processing system. Mary Ann Cahill corrected the English.

Correspondence should be addressed to Claudia A. O. Stuermer, Faculty of Biology, University of Konstanz, D-78434 Konstanz, Germany.

Copyright (C) 1996 Society for Neuroscience $0270-6474 / 96 / 165741-08 \$ 05.00 / 0$ which contribute to the formation of proper myelin sheath. MAG has recently been discussed as another inhibitor of axon growth (McKerracher et al., 1994; Mukhopadhyay et al., 1994).

Oligodendrocytes develop from O-2A progenitor cells under the influence of a variety of growth factors (Raff et al., 1983). Some, such as interleukin-6 (Il-6), ciliary neurotrophic factor (CNTF), and insulin-like growth factor (IGF), enhance oligodendrocyte differentiation and promote their survival (Barres et al., 1993). Platelet-derived growth factor (PDGF), another important growth factor with three subtypes (PDGF-AA, PDGF-AB, and PDGF-BB) (Heldin et al., 1988), stimulates proliferation of O-2A progenitors (Noble et al., 1988; Richardson et al., 1988), positively influences their survival (Barres et al., 1993), and contributes to the expression of myelin proteins (Grinspan et al., 1993).

An additional, unexpected function of PDGF that affects the inhibitory properties of oligodendrocytes during their differentiation in vitro is described here. Oligodendrocytes that remained in medium containing PDGF-AB for more than 1 week lost their inhibitory influence on growth cones. Moreover, IN-1 immunoreactivity was significantly reduced in PDGF-treated oligodendrocytes, whereas expression of O1/GalC, MBP, PLP, and MAG remained unchanged as judged from immunostaining experiments with the relevant antibodies. Oligodendrocytes regained their inhibitory influence when deprived of PDGF-AB. Thus, PDGF-AB appears to regulate-directly or indirectly-the expression of the NI.

\section{MATERIALS AND METHODS}

Primary cultures from rat optic nerves. Mixed glial cultures from optic nerves of 7- to 10-d-old rats were prepared as described (Schwab and Caroni, 1988). Cells were plated onto polylysine/laminin-coated coverslips at low density $\left(\sim 1000\right.$ cells $\left./ \mathrm{cm}^{2}\right)$ and kept for $8-10 \mathrm{~d}$ in either 
Dulbecco's minimal essential medium (DMEM) or in DMEM/Ham's F12 (1:1). Both media contained $10 \mu \mathrm{g} / \mathrm{ml}$ bovine serum albumin, $10 \mu \mathrm{g} / \mathrm{ml}$ human transferrin, $5 \mu \mathrm{g} / \mathrm{ml}$ bovine insulin, $20 \mathrm{nM}$ progesterone, $30 \mathrm{nM}$ Na-selenite, $100 \mu \mathrm{M}$ putrescine, $15 \mathrm{~nm}$ tri-iodothyronine, $2 \mathrm{~mm}$ glutamine, $1 \mathrm{~mm}$ Na-pyruvate, $0.4 \%$ methyl cellulose (MC), and $50 \mu \mathrm{g} / \mathrm{ml}$ gentamycin. Three times a week half of the medium was changed. Recombinant PDGF (PDGF-AB; Sigma, Deisenhofen, Germany or Biomol, Hamburg, Germany; $10 \mathrm{ng} / \mathrm{ml}$ ) was added to the cultures three times a week.

Secondary cultures enriched in oligodendrocytes and astrocytes. Mixed glial cultures were prepared by mechanical dissociation of cerebral cortices of neonatal rat pups. Cells were grown to confluency in DMEM containing $10 \%$ fetal calf serum (FCS) in polylysine-coated tissue culture flasks. Oligodendrocytes were collected according to methods described earlier (McCarthy and De Vellis, 1980) and replated onto polylysine/ laminin-coated coverslips at a density of $\sim 1000$ cells $/ \mathrm{cm}^{2}$ in defined DMEM or DMEM/F12 medium with supplements, described above.

Cultures from which most of the oligodendrocytes were removed, (and which were thus enriched in astrocytes) were used to collect astrocyteconditioned serum-free medium. This medium was applied to oligodendrocytes in one experiment instead of PDGF.

Cocultures of oligodendrocytes and neuronal explants. Dorsal root ganglia (DRGs) from neonatal rats were chopped into small segments, resuspended in DMEM/F12 containing $100 \mathrm{ng} / \mathrm{ml}$ nerve growth factor (NGF) and $0.4 \% \mathrm{MC}$, and added to glial cultures that had been rinsed three times in PDGF-free culture medium before the addition of DRGs. Cocultures in Petriperm dishes (Heraeus, Hanau, Germany) were incubated in a small volume of medium overnight for DRG attachment and neurite outgrowth. These cultures were used either for time-lapse videomicroscopy, or, after an incubation period of $2 \mathrm{~d}$, subjected to a double immunostaining procedure to visualize oligodendrocytes and to identify DRG neurites.

In some experiments, the antimitotic drug cytosine arabinofuranoside (AraC; Sigma) was added $(8 \mu \mathrm{M})$ to glial cultures for at least $3 \mathrm{~d}$ before the addition of DRG explants to prevent the emergence of new (immature) oligodendrocytes from progenitor cells and to suppress astrocyte proliferation.

Explants of goldfish and Xenopus retinae were obtained from adult animals that had received optic nerve cuts $10-14 \mathrm{~d}$ before preparation. The retinae were attached to nylon filters, cut into $300 \mu \mathrm{m}$ wide stripes, and placed, ganglion cell layer down, onto the coverslips carrying the glial cells. Cocultures were maintained overnight at $28^{\circ} \mathrm{C}$, a compromise temperature that allows survival of rat oligodendrocytes and outgrowth of fish and amphibian axons (Bastmeyer et al., 1993; Lang et al., 1995).

Time-lapse videomicroscopy. Cocultures of glial cells and neuronal explants were transferred to DMEM/F12 containing $100 \mathrm{ng} / \mathrm{ml} \mathrm{NGF}$, $0.4 \% \mathrm{MC}$, and $15 \mathrm{mM}$ HEPES buffer (instead of bicarbonate) and left to equilibrate for 1 hour on the heatable stage of an inverted microscope (Zeiss, Oberkochen, Germany). Cocultures with rat DRGs were kept at $37^{\circ} \mathrm{C}$. For the cocultures with fish and amphibian axons, stage temperature was reduced to $28^{\circ} \mathrm{C}$. Time-lapse sequences of axons encountering oligodendrocytes were videotaped over 12-16 hr. At least three cocultures were examined in each experiment. Only those oligodendrocytes that displayed a highly branched morphology indicating complete differentiation were chosen for the recordings. In most cases, living oligodendrocytes were stained with monoclonal antibodies (mAbs) O1 (Sommer and Schachner, 1981) or anti-GalC (Ranscht et al., 1982) before the time-lapse recordings to facilitate identification of differentiated cells.

Immunofluorescence and quantification of staining intensity. Monoclonal antibody (mAb) A2B5, a marker for O-2A progenitor cells (Raff et al., 1983), and $\mathrm{mAbs}$ anti-GalC and O1, markers for differentiated oligodendrocytes (Sommer and Schachner, 1981; Ranscht et al., 1982), were applied to live cells as described (Bastmeyer et al., 1991). Cocultures of oligodendrocytes and DRG neurons were double-labeled with mAb O1 and a rabbit antiserum to the cell adhesion molecule L1 (gift of F. Rathjen, MDC, Berlin, Germany). To detect NI on the cell surface, live oligodendrocytes were incubated $\left(2 \mathrm{hr} ; 37^{\circ} \mathrm{C}\right)$ with undiluted hybridoma supernatant containing $\mathrm{mAb}$ IN-1 (Caroni and Schwab, 1988b). For detection of myelin markers expressed by oligodendrocytes, double and triple fluorescence labeling was performed with an mAb against MBP (dilution 1:10), a rabbit antiserum against PLP (dilution 1:200; both gifts of C. Linington, MPJ, Munich, Germany), and a rabbit antiserum to MAG (dilution 1:200; gift of $\mathrm{M}$. Schachner, ETH, Zürich, Switzerland) after fixation in methanol (5 min; $-20^{\circ} \mathrm{C}$ ) and $4 \%$ paraformaldehyde $(5 \mathrm{~min}$; room temperature). In some cases, for exclusive labeling of MAG expressed on the cell surface, live oligodendrocytes were incubated with the antiserum $\left(1 \mathrm{hr} ; 37^{\circ} \mathrm{C}\right)$. Secondary antibodies were goat anti-mouse 7-amino-4-methylcoumarin-3-acetate, goat anti-rat tetramethylrhodamine-isothiocyanate (TRITC), and goat anti-rabbit TRITC or fluorescein-isothiocyanate (all from Dianova, Hamburg, Germany; dilution 1:200). For visualization of bound IN-1 antibodies, $\mu$-chainspecific donkey or rat anti-mouse cyanin-3 (Cy-3; Dianova; dilution 1:1000) was used.

The intensity of immunofluorescence was quantified in oligodendrocytes double-labeled with mAb IN-1 against NI and anti-PLP or antiMAG to see whether the PDGF-induced reduction of inhibitory substrate properties is paralleled by a reduction of IN-1 immunoreactivity. Fluorescence values were measured in the flat membraneous extensions typical of differentiated oligodendrocytes, which were visualized by PLP or MAG immunoreactivity. The staining procedure with the Cy-3conjugated secondary antibody produced nonspecific fluorescence in the soma but not on the membranous extensions (see Fig. 7).

Moreover, when the intensity measurements were adapted to the weak immunofluorescence signals obtained with IN-1 and secondary antibody, the soma of the oligodendrocytes produced a signal even when no antibody was applied. This signal from the soma was increased when cells were subjected to staining with Cy-3-conjugated secondary antibody alone (see Fig. 7). Therefore, control experiments were performed in which PDGF-treated and -untreated oligodendrocytes $(n=12$ each) were subjected to the same immunofluorescence intensity measurements after exposure to $\mathrm{Cy}-3$-conjugated secondary antibody alone. More than $80 \%$ of the immunofluorescence of the somata turned out to be unspecific. Therefore, cell bodies of oligodendrocytes were not included in the evaluation.

The level of unspecific Cy-3 fluorescence intensity on the membraneous extensions, however, was insignificant compared with that produced by primary and secondary antibodies (see Fig. 7). In each measurement, the fluorescence intensity was averaged over a membrane area between 25 and $300 \mu \mathrm{m}^{2}$. Six measurements were made per oligodendrocyte, and values were obtained from 12 cells in each experimental group (see Fig. 7) representing one set. Three sets of experiments, including controls, were performed, two sets with anti-PLP as reference and one set with anti-MAG. Figure 7 represents the results of one set. Fluorescence images and measurements were taken with an ICCD camera (Hamamatsu, Herrsching, Germany) and quantified using the image processing system HPD-CPx (Hamamatsu) equipped with the Argus software.

To detect glial cells undergoing DNA replication, cultures were incubated in medium with $10 \mu \mathrm{M}$ bromodeoxyuridine (BrdU; Sigma). Twentyfour hours later, the live cultures were stained with mAb 01, exposed to goat anti-mouse TRITC and subsequently processed for anti-BrdU immunohistochemistry as described elsewhere (Nowakowski et al., 1989).

\section{RESULTS \\ PDGF-induced reduction of inhibitory properties of oligodendrocytes}

Control and experimental glial cell cultures were maintained 8-10 $\mathrm{d}$ in medium known to enhance oligodendrocyte differentiation (Raff et al., 1985; Noble et al., 1988; Grinspan et al., 1993), but only the experimental cultures received PDGF. In both cultures, the majority of cells acquired a highly branched morphology typical of differentiated oligodendrocytes (Fig. 1). As expected, these were more numerous in the PDGF-treated cultures than in controls (Noble et al., 1988; Richardson et al., 1988).

Retina explants or DRGs were cocultured with oligodendrocytes. In control cultures, the vast majority of axons extending from the neuronal tissue avoided crossing the oligodendrocytes (Fig. 1). This is typical for most neurons tested so far (Fawcett et al., 1989; Bandtlow et al., 1990; Bastmeyer et al., 1991; Lang et al., $1995)$ and is indicative of the inhibitory properties of differentiated rat oligodendrocytes. However, in PDGF-containing cultures the opposite was observed. The majority of axons grew over the oligodendrocytes (Fig. 1).

To examine the reaction of individual growth cones when they contact these oligodendrocytes, their encounters were monitored with time-lapse video recordings. With DRGs added to 8- to 10 -d-old control cultures, 27 of 34 contacts resulted in growth 

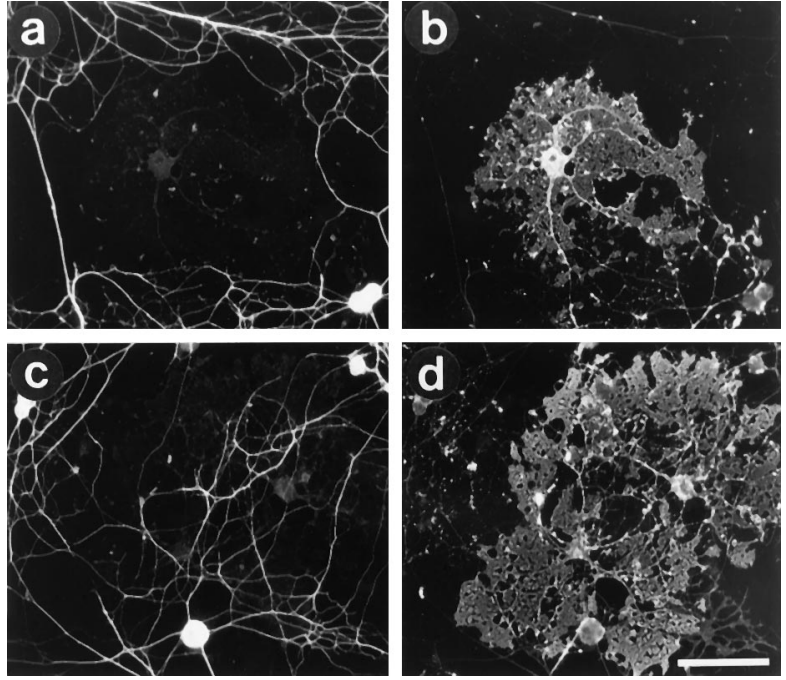

Figure 1. Cocultures of oligodendrocytes and DRG neurons doublestained with anti-L1 $(a, c)$ and O1 $(b, d)$. DRG neurites in PDGF-free control medium avoid crossing the oligodendrocytes $(a, b)$. PDGF-treated oligodendrocytes, however, are freely crossed by the neurites $(c, d)$. Scale bar, $50 \mu \mathrm{m}$.

cone collapse and subsequent axonal retraction; seven growth cones avoided the oligodendrocytes by growing around the cells (Fig. 2). In contrast, the majority of oligodendrocytes raised in PDGF-containing medium for 8-10 d (henceforth called PDGFtreated oligodendrocytes) failed to induce DRG growth cone collapse (Fig. 2). In 32 of 49 encounters, growth cones managed to cross the oligodendrocytes. Only nine resulted in growth cone collapse, and in eight events axons grew around the cells (Fig. 2). All examined oligodendrocytes exhibited a highly branched morphology and were O1-positive, like their untreated counterparts (Fig. 3).

A similar change in growth cone behavior was seen when fish

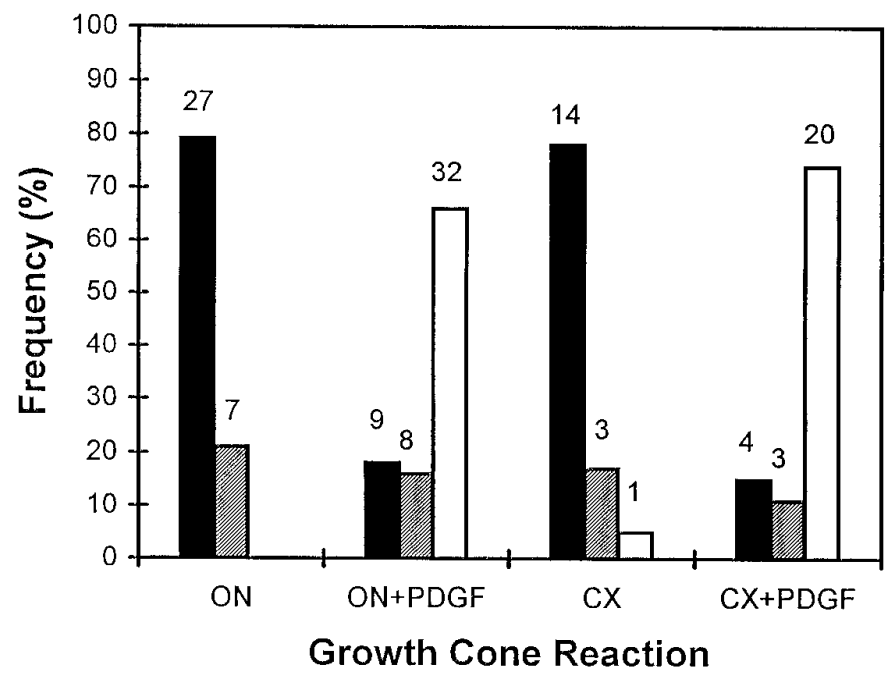

Figure 2. Quantification (in percentage) of DRG growth cone reactions after contacting oligodendrocytes. Black bars represent collapse, hatched bars growth around the cell, and white bars growth across the cell. Numbers of growth cones are given on top of each bar. Oligodendrocytes were derived from the optic nerve and cortex, respectively, and raised over 8-10 $\mathrm{d}$ in medium without PDGF $(O N, C X)$ or in medium with PDGF $(O N+P D G F, C X+P D G F)$ before the addition of DRG neurons. and Xenopus retinal axons were confronted with PDGF-treated oligodendrocytes (Fig. 3). In control cultures, most growth cones of both fish (Bastmeyer et al., 1991) and Xenopus retinae collapse after contact with rat oligodendrocytes. With PDGF-treated oligodendrocytes, however, the majority of Xenopus as well as goldfish retinal axons crossed the cells (Fig. 3). Again, both PDGFtreated oligodendrocytes and oligodendrocytes in control cultures were positive for $\mathrm{O} 1$ and $\mathrm{GalC}$, respectively.

In summary, the majority of growth cones of all three neuron types collapse in contact with oligodendrocytes of control cultures but cross PDGF-treated oligodendrocytes. This suggests that the 8-10 d exposure to PDGF alters the inhibitory substrate properties of oligodendrocytes.

To determine whether oligodendrocytes from other areas of the CNS similarly fail to elicit growth cone collapse when treated with PDGF, glial cells were obtained from rat cortices. Again, cultures were raised for 8-10 d-one group maintained in medium with PDGF, the control group in medium without PDGF.

Oligodendrocytes in control cultures elicited DRG growth cone collapse in 14 of 18 encounters (Fig. 2). One growth cone grew onto an oligodendrocyte but collapsed after a few minutes and retracted. Three growth cones avoided the oligodendrocytes. PDGF-treated oligodendrocytes, however, were crossed by growth cones in the majority of cases, i.e., in 20 of 27 (Fig. 2). Only three growth cones avoided the oligodendrocytes and four collapsed. In two instances, growth cones extended for considerable distance onto an oligodendrocyte, but then collapsed and retracted.

PDGF is known to be mitogenic for oligodendrocyte precursor cells (Noble et al., 1988; Richardson et al., 1988). To exclude the possibility that PDGF-treated cultures might contain large numbers of newly differentiated oligodendrocytes, which may not yet have acquired their inhibitory substrate properties, the oligodendrocyte cultures received the anti-mitotic drug AraC. AraC was added to cultures that grew in the absence or presence of PDGF, and was present for at least $3 \mathrm{~d}$ before addition of neuronal explants.

This procedure resulted in the elimination of proliferating cells but left behind the differentiated, highly branched O1/GalCpositive oligodendrocytes. The efficiency of the AraC treatment was evaluated by BrdU incorporation assays in parallel cultures, which were devoid of BrdU-labeled cells. In cocultures with DRG neurons, the numbers of oligodendrocytes from AraC/PDGFtreated cultures, which were avoided or overgrown by neurites, were counted (Fig. 4) and compared with cocultures with AraCtreated oligodendrocytes not having received PDGF. In PDGFtreated cultures, $>80 \%$ of the oligodendrocytes were overgrown by DRG neurites, and the majority $(70 \%)$ of cells were avoided in the controls. This is consistent with the outcome of the time-lapse experiments (Fig. 2).

\section{Expression of advanced myelin marker proteins and IN-1 immunoreactivity by PDGF-treated oligodendrocytes}

Although the substrate properties of O1/GalC-positive highly branched oligodendrocytes in PDGF-treated oligodendrocytes differed from those of oligodendrocytes in untreated cultures, the proportion of O1/GalC-positive cells was identical in both cultures. More than $90 \%$ of the highly branched oligodendrocytes expressed $\mathrm{O} 1 / \mathrm{GalC}$ after 8-10 d, in PDGF-treated as well as in untreated cultures. Oligodendrocytes of both groups were negative for the early differentiation marker A2B5 (Raff et al., 1983). Furthermore, O1/GalC-positive oligodendrocytes did not incor- 

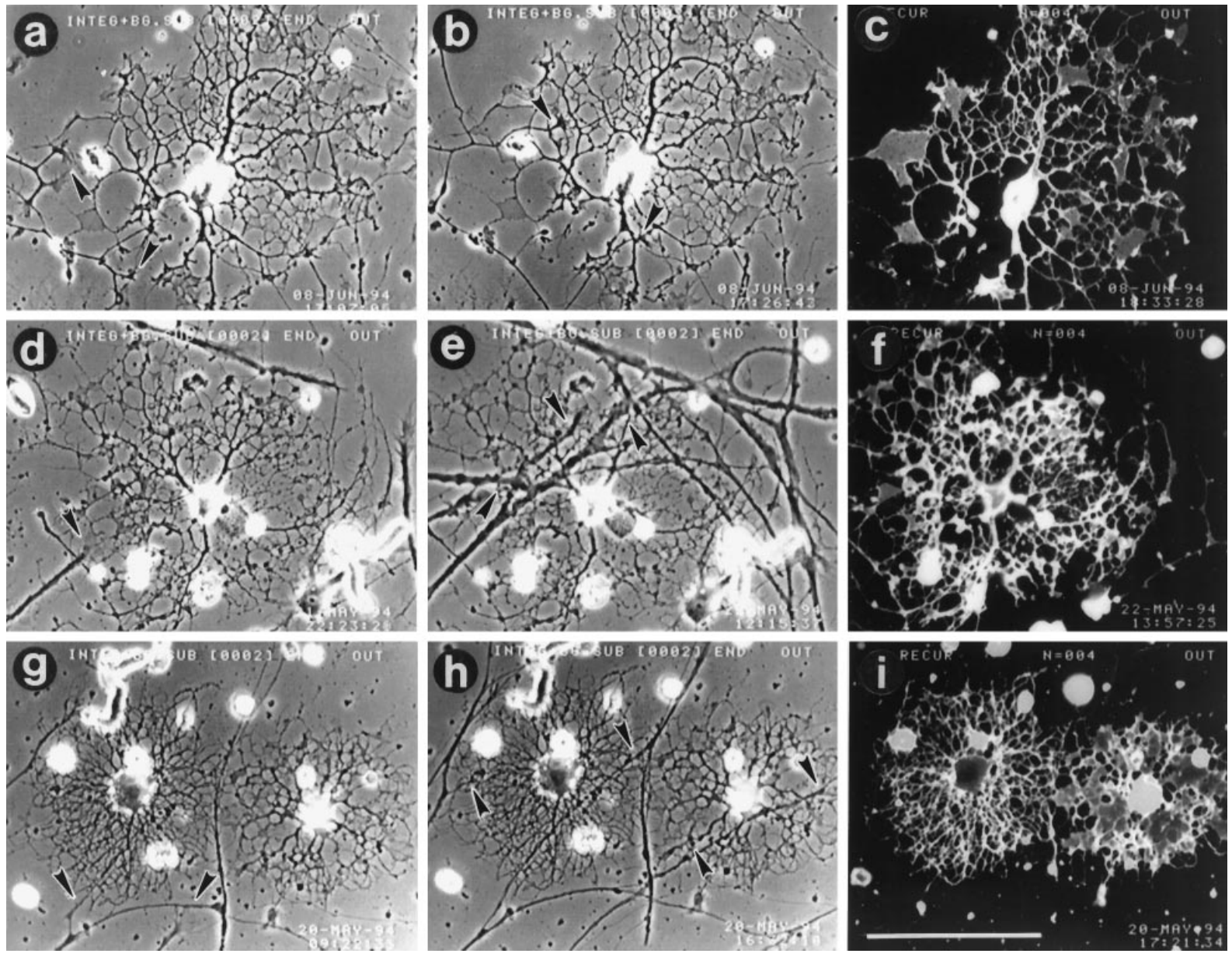

Figure 3. Selected images of time-lapse movies illustrating the response of growth cones after encountering PDGF-treated oligodendrocytes, which are O1-positive $(c, f, i) . a, b$, DRG growth cones. $d, e$, Xenopus retinal growth cones. $g, h$, Goldfish retinal growth cones. Growth cones (marked by arrowheads) cross the oligodendrocytes. Scale bar, $100 \mu \mathrm{m}$.

porate BrdU, either in cultures treated with PDGF or in untreated cultures. Moreover, when exposed to antibodies against advanced myelin markers (MBP, PLP, MAG), highly branched oligodendrocytes also exhibited MBP and PLP immunoreactivity, whether raised with PDGF or without (Fig. 5).

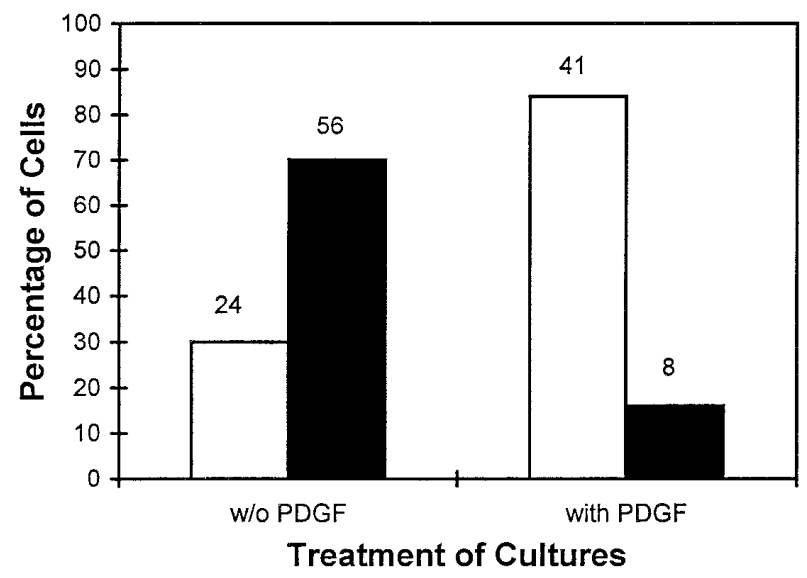

Figure 4. Percentage of AraC-treated oligodendrocytes cultured in the absence (w/o PDGF) or presence of PDGF (with PDGF) that were avoided (black bars) or crossed (white bars) by DRG neurites. The number of cells is indicated on top of each bar.
These oligodendrocytes also possessed intense MAG immunoreactivity. This is interesting because MAG was recently described as being inhibitory to adult DRGs and other axons (McKerracher et al., 1994; Mukhopadhyay et al., 1994). But here, with MAG present on PDGF-treated oligodendrocytes, all three types of growth cones grew, in the majority of cases (Fig. 2, 3), across the cells.

Using a highly sensitive detection method with Cy-3-conjugated secondary antibodies in conjunction with a computer-assisted quantification procedure, the intensity of IN-1 immunoreactivity in membraneous extensions of highly branched oligodendrocytes was measured. Three independent sets of experiments, including the appropriate controls (see Materials and Methods) were performed, and all three gave similar results. The outcome of one set is shown in Figure 7. The intensity of IN-1 immunofluorescence was reduced in PDGF-treated oligodendrocytes, representing $\sim 30 \%$ of that seen in untreated control cultures (Fig. 6, 7). These oligodendrocytes, PDGF-treated and controls, were doublestained with antibodies against PLP or MAG. PLP immunoreactivity was strong in both sets of oligodendrocytes and appeared slightly increased in the PDGF-treated cells. The same applied to MAG immunoreactivity (not shown). Furthermore, there was no direct correlation between the intensities of IN-1 and PLP immunofluorescence for individual oligodendrocytes (correlation coefficient $r=0.04$ and 0.11 for PDGF-treated and untreated cells, 

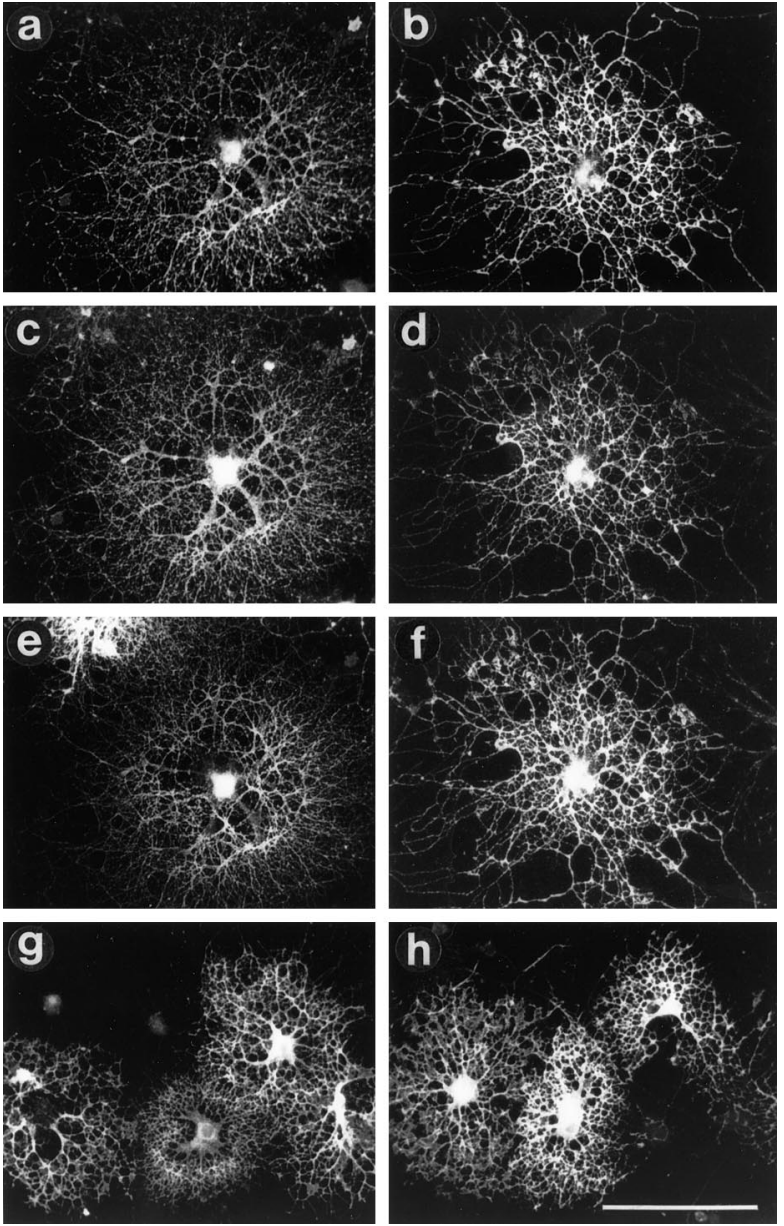

Figure 5. Oligodendrocytes whether raised in medium with $(a, c, e, g)$ or without $(b, d, f, h) \mathrm{PDGF}$, are immunopositive after exposure to mAb O1 $(a, b)$, anti-MBP $(c, d)$, anti-PLP $(e, f)$, and anti-MAG $(g, h)$. Scale bars: $50 \mu \mathrm{m}$ in $a-f ; 100 \mu \mathrm{m}$ in $g$ and $h$.

respectively). The same measurements performed on cortical oligodendrocytes yielded identical results (not shown).

These data show that PDGF treatment of oligodendrocytes does not change the expression of advanced myelin marker proteins, and thus does not prevent their normal in vitro differentiation. However, PDGF does influence and reduces IN-1 immunoreactivity and the inhibitory properties of oligodendrocytes, most likely by downregulating the production or expression of NI on the oligodendrocyte's surface.

\section{The effect of PDGF on substrate properties of oligodendrocytes is reversible}

Whether exposure to PDGF could change the substrate properties of oligodendrocytes that had already become inhibitory was tested in a further group of experiments.

As demonstrated earlier and confirmed here, virtually all oligodendrocytes in control medium differentiated and had inhibitory substrate properties (Figs. 1, 2). However, when these oligodendrocytes were then exposed to medium with PDGF for another week, most of them became growth-permissive (Fig. 8). This was concluded from the behavior of DRGs that were added to the culture at this time. Of the 22 encounters between oligodendrocytes and DRG growth cones observed here, only four resulted in growth cone collapse. Three axons avoided the oligo-
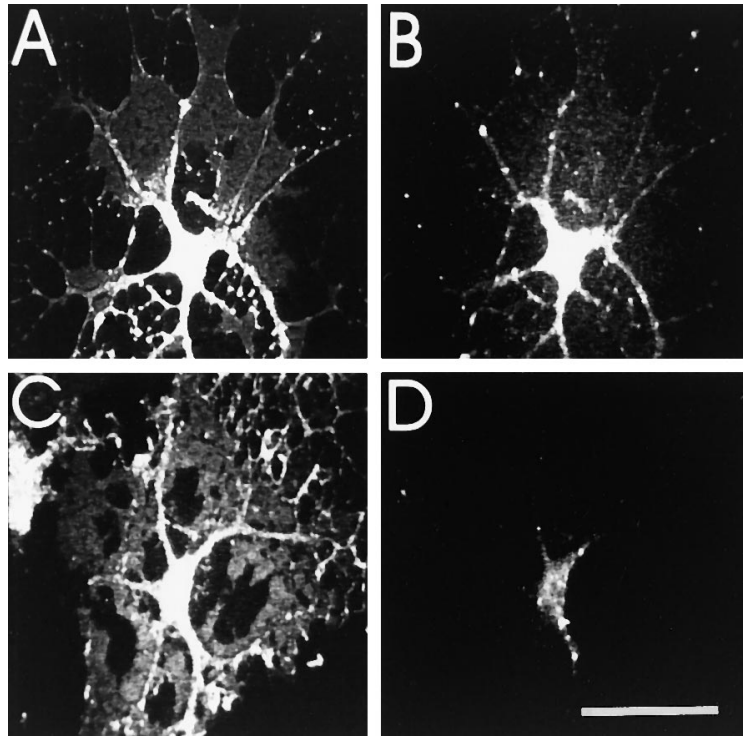

Figure 6. Double labeling of oligodendrocytes with anti-PLP $(A, C)$ and mAb IN-1 $(B, D)$. In both PDGF-treated $(C, D)$ and control cultures $(A$, $B$ ), oligodendrocytes possess membraneous extensions with intense PLP immunoreactivity. IN-1 immunoreactivity, although readily detectable in membraneous extensions of untreated oligodendrocytes $(B)$, is weak in PDGF-treated cells $(D)$ and basically absent from their membraneous extensions. More than $80 \%$ of staining on the soma results from the secondary Cy-3-conjugated antibody alone. Images of IN-1immunoreactive oligodendrocytes are enhanced. Scale bar, $50 \mu \mathrm{m}$.

dendrocytes, but 15 traversed the cells. Thus, it appears that oligodendrocytes that had acquired inhibitory substrate properties lose them again through the influence of PDGF.

Oligodendrocytes were cultured in medium containing PDGF for 1 week and then kept in medium without PDGF for an additional $10 \mathrm{~d}$ to test whether absence of PDGF would allow the permissive oligodendrocytes to acquire their normal inhibitory properties. When DRGs were added, their growth cones collapsed in 11 of 20 encounters and six avoided the cells. Only three managed to cross them (Fig. 8). This indicates that rat oligodendrocytes reacquire their inhibitory substrate properties in the absence of PDGF.

The reappearance of inhibitory substrate properties after withdrawal of PDGF was slow. A large proportion (60\%) of oligodendrocytes that had been raised $8 \mathrm{~d}$ in PDGF and then kept for an additional $6 \mathrm{~d}$ in PDGF-free medium were still growth-permissive. Seventeen growth cones crossed the cells; nine collapsed and two grew around the cell.

\section{Effect of glial cell-conditioned medium}

It has been reported that DRG axons are not impaired in their growth by oligodendrocytes, when these oligodendrocytes reside on type I astrocytes (Fawcett et al., 1992). Because type I astrocytes are known to produce PDGF (Richardson et al., 1988), we tested the effect on oligodendrocytes of medium conditioned by glial cell cultures in which most cells were astrocytes.

Oligodendrocytes were prepared as described in the previous experiments, but now received conditioned medium (CM) without any additional growth factor. After $8 \mathrm{~d}$, DRGs were added. Much as with PDGF-treated oligodendrocytes, the majority of growth cones grew onto and over CM-treated oligodendrocytes. Eight growth cones crossed, two avoided the cells, and only one collapsed (Fig. 8). 


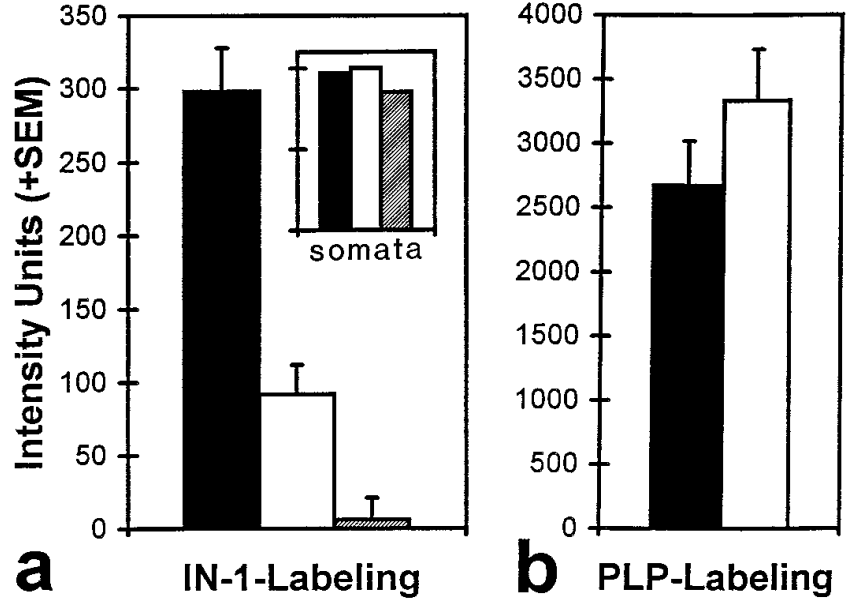

Figure 7. Comparison of labeling intensity in oligodendrocytes exposed to mAb IN-1 (a) and anti-PLP $(b)$. Measurements were taken in membrane sheets of double-labeled oligodendrocytes from control (black bars) and PDGF-treated cultures (white bars). Hatched bars in a represent background staining on the membraneous extensions of oligodendrocytes generated by the secondary Cy-3-conjugated antibody alone. The insert in $a$ represents intensity measurements (in percentage) of the somata. Black bar in insert represents somata of control oligodendrocytes; white bar, PDGF-treated oligodendrocytes (100\%); hatched bar, Cy-3-conjugated antibody alone. Intensity units were generated by the image processing system. On average, IN-1 labeling in membrane sheets is decreased by $70 \%$ in PDGF-treated oligodendrocytes, whereas PLP labeling remains constant or increases. Note that the intensity of anti-PLP staining is approximately 10 times that of $\mathrm{mAb}$ IN-1.

Thus, media conditioned by mixed glial cell cultures rich in astrocytes alter the substrate properties of oligodendrocytes much like recombinant PDGF-AB, and perhaps through the action of PDGF produced by astrocytes.

\section{DISCUSSION}

The preceding results demonstrate that exposure to PDGF provokes a marked reduction of the well known inhibitory substrate properties (Schwab et al., 1993) in a significant proportion of oligodendrocytes. It is therefore suggested that PDGF acts on regulatory pathways for the expression of NI associated with CNS myelin and oligodendrocytes. This suggestion is based on evaluation of oligodendrocyte substrate properties revealed by the reaction of growth cones, and on a quantitative assessment of immunoreactivity with antibody IN-1 against the neurite growth inhibitory proteins (Caroni and Schwab, 1988b).

Quantification of growth cone responses demonstrates that growing across oligodendrocytes instead of collapsing is predominant when oligodendrocytes have been exposed to PDGF for a certain length of time. Otherwise, all three types of neurons used here exhibit growth cone collapse as the typical response when their growing tip encounters oligodendrocytes, as shown in earlier studies (Fawcett et al., 1989; Bandtlow et al., 1990; Bastmeyer et al., 1991) and the present control experiments. Because PDGF was the only component that was varied between experimental and control cultures, the observed differences in substrate properties of oligodendrocytes in experimental and control cultures must result from the (direct or indirect) action of this growth factor.

NI are potent inducers of growth cone collapse. Neutralization of these inhibitors by the antibody IN-1 (Caroni and Schwab, 1988 b) results in a dramatic increase in axon growth across oli-

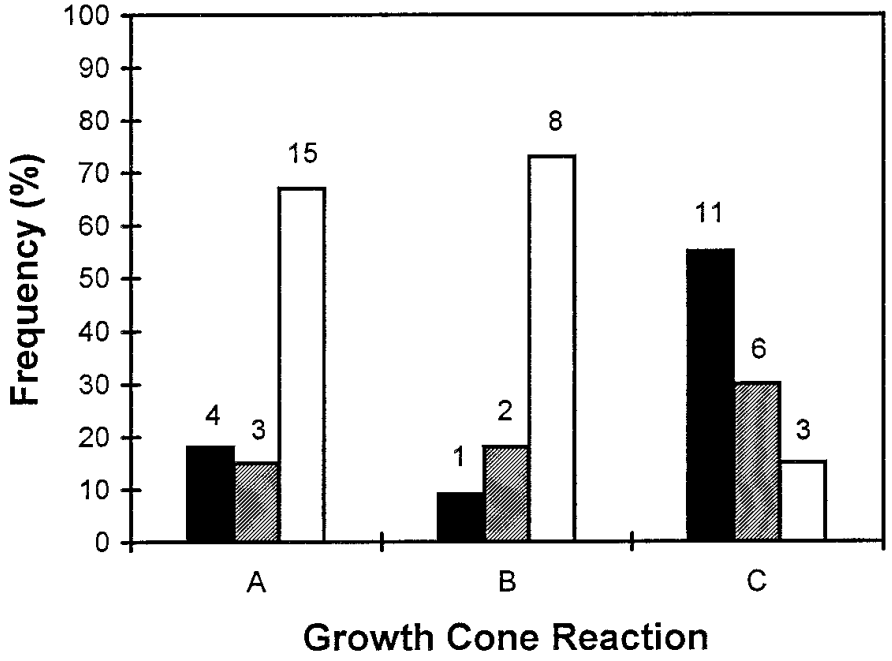

Figure 8. Quantification (in percentage) of growth cone reactions after contact with oligodendrocytes that were first raised 8-10 d in medium without PDGF and then transferred to medium containing $\operatorname{PDGF}(A)$ or astrocyte-conditioned medium $(B)$. In $C$, oligodendrocytes were raised in medium with PDGF and then transferred to medium without PDGF. Growth cone responses were monitored $8 \mathrm{~d}$ after the transfer of the oligodendrocytes. Black bars, Collapse; hatched bars, growth around the cell; white bars, growth across the cell. The number of growth cones is given on top of each bar.

godendrocytes (Bandtlow et al., 1990; Bastmeyer et al., 1991), much as is the case with PDGF-treated oligodendrocytes in this study. This suggests that PDGF indeed affects the growth inhibitors-most likely through interference with their expression. This view is supported by the fact that the intensity of IN-1 immunoreactivity was markedly decreased in PDGF-treated oligodendrocytes. It is unlikely, although not excluded by the present experiments, that PDGF influences the inhibitors by modifying their structure or functional epitopes.

The reappearance of inhibitory substrate properties of PDGFtreated oligodendrocytes after PDGF withdrawal, as evaluated by growth cone reactions, was unusually slow. However, NI proteins are not cloned to date, and the events on the second-messenger level responsible for the prolonged time course of the action of PDGF on NI expression remain to be elucidated.

We favor the view that NI, rather than other known surface molecules with nonpermissive functions, are regulated by PDGF. First, the similarity of axon responses to oligodendrocytes treated with IN-1 (Bandtlow et al., 1990; Bastmeyer et al., 1991) and PDGF is striking. Second, IN-1 immunoreactivity decreases, whereas the expression of the other putative inhibitory molecule, MAG (McKerracher et al., 1994; Mukhopadhyay et al., 1994), remains high or even increases in PDGF-treated oligodendrocytes. Moreover, MAG, while affecting certain neurons including adult DRGs, does not inhibit growth of newborn rat DRG neurons (Johnson et al., 1989; Bartsch et al., 1995), which were used in this study. Whether MAG negatively influences the growth of fish and Xenopus retinal ganglion cell axons has not been tested in the relevant earlier studies, but in the present experiments, MAG associated with oligodendrocytes did not prevent fish or Xenopus retinal ganglion cell axons from crossing these cells. Third, Janusin, another surface molecule potentially contributing to the nonpermissive substrate properties of oligodendrocytes, does not cause growth cone collapse as consistently as the inhibitors (Taylor et al., 1993). Instead, purified Janusin applied in spots to 
coverslips causes axons to avoid the protein-containing regions by turning away from them. The few remaining collapse and avoidance responses seen with IN-1-treated and PDGF-treated oligodendrocytes, however, could perhaps be attributed to these or other molecules, or to incomplete abolition of the inhibitors.

Because oligodendrocytes exhibit their inhibitory properties after differentiation to highly branched, O1/GalC-expressing cells, care was taken to monitor only growth cone contacts with differentiated cells. In both PDGF-treated and untreated glial cultures, the vast majority of oligodendrocytes were indeed $01 / \mathrm{GalC}$ positive at the time of the recordings. Also, none of the cells under consideration had arisen recently through proliferation of progenitors. This was demonstrated by experiments with the cytostatic drug AraC, which prevented the development of new oligodendrocytes from dividing precursor cells, and by BrdU incorporation assays. More importantly, labeling of oligodendrocytes with specific antibodies against advanced myelin marker proteins confirmed that PDGF had not prevented differentiation and myelin protein expression in these glial cells. Thus, the low proportion of inhibitory oligodendrocytes in PDGF-treated cultures is not attributable to incomplete differentiation of the cells or to continued generation of "young" oligodendrocytes from proliferating precursors. Moreover, differentiated oligodendrocytes, the majority of which caused growth cone collapse in controls, had lost their inhibitory properties after $8-10 \mathrm{~d}$ in PDGF-containing medium. This substantiates the suggestion that PDGF causes a downregulation of the NI in mature oligodendrocytes, and that PDGF withdrawal has the opposite effect.

Another possibility that could also account for the increase in axon growth across oligodendrocytes is an abnormal distribution of NI in PDGF-treated oligodendrocytes, such that the cells fail to translocate the NI proteins to their membraneous extensions. This is derived from the observation that IN-1 immunofluorescence was present in the cell bodies and soma near processes of PDGFtreated oligodendrocytes. Most of this fluorescence, however, represents an artifact caused from autofluorescence and by the immunostaining procedure with the Cy-3-conjugated secondary antibodies. Still, both views, downregulation of the expression or prevention of the normal distribution of NI, are compatible with the outcome of the present functional assays.

Because mAb IN-1 does not reliably recognize its antigen in immunoblots (producing more than 20 bands) and probes for in situ hybridization are not available, more specific tests of NI expression in PDGF-treated and untreated oligodendrocytes cannot be performed at present.

The observation that conditioned media had similar effects as recombinant PDGF on the increase in permissiveness of oligodendrocytes is interesting in light of earlier findings. There was no inhibition of axon growth in complex glial cultures in which oligodendrocytes resided on beds of astrocytes (Fawcett et al., 1992). With the notion that astrocytes secrete PDGF (Richardson et al., 1988; Pringle et al., 1989), this very growth factor may have affected the normal expression of inhibitors on these oligodendrocytes. Our attempts to deplete media of PDGF in such cultures using commercially available PDGF antibodies failed, so a direct test of the above speculation is missing.

Influences of PDGF on oligodendrocytes requires the presence of PDGF receptors, which are known to activate intracellular signaling cascades and gene expression (Rozengurt, 1986; Hart et al., 1989a). It has been demonstrated that oligodendrocyte precursors express PDGF receptors of the $\alpha$-subtype (Hart et al., 1989b; McKinnon et al., 1990). Using the relevant antibodies (kindly provided by M. Paech, Roche, Basel, Switzerland), we were able to confirm the presence of $\alpha$-receptors on oligodendrocyte precursors; they were not detected on differentiating oligodendrocytes whether treated with PDGF or not (D. Lang and M. Hille, unpublished observations). PDGF $\alpha$-receptors are activated by all three forms of PDGF, PDGF-AA, PDGF-BB, and PDGF-AB (Barres et al., 1993), the last of which was the form used in this study.

PDGF (all forms) was shown to promote proliferation of precursors (Noble et al., 1988; Richardson et al., 1988), and PDGF-AA supports their survival, but lower concentrations are needed for the survival effect (Barres et al., 1993). From this dual effect it was proposed that more than one type of receptor and diverse signaling cascades are involved (Barres et al., 1993). These two effects were proposed to be evoked by PDGF $\alpha$-receptors and by a receptor that remains to be identified. Survival of oligodendrocytes was suggested to be promoted by this novel receptor type (Barres et al., 1993).

Whether the effect of PDGF-AB on oligodendrocyte substrate properties seen here rests on the activation of the receptor that promotes survival is unknown. The PDGF effects noted in the present study, together with the beneficial effect of PDGF described earlier (Grinspan et al., 1993) on increased production of mRNAs for myelin marker proteins, speaks for a more longlasting presence of the relevant PDGF receptor or mediation of the effects through pathways that do not depend on PDGF receptors.

Whether PDGF would have beneficial effects on axonal regeneration in vivo is not known. One might assume that after CNS injury, blood platelets release PDGF in large quantities, which might render oligodendrocytes near the lesion site permissive for axonal regrowth. However, inhibitors associated with myelin (Schwab and Caroni, 1988) in these regions may persist and continue to exert their negative effects on regrowing axons. Still, to our knowledge this is the first account of the regulation of oligodendrocyte inhibitors, and this may allow assessment of regulatory pathways.

\section{REFERENCES}

Bandtlow C, Zachleder T, Schwab ME (1990) Oligodendrocytes arrest neurite growth by contact inhibition. J Neurosci 10:3837-3848.

Barres BA, Schmid R, Sendtner M, Raff MC (1993) Multiple extracellular signals are required for long-term oligodendrocyte survival. Development 118:283-295.

Bartsch U, Bandtlow CE, Schnell L, Bartsch S, Spillmann AA, Rubin BP, Hillenbrandt R, Montag D, Schwab ME, Schachner M (1995) Lack of evidence that myelin-associated glycoprotein is a major inhibitor of axonal regeneration in the CNS. Neuron 15:1375-1381.

Bastmeyer M, Beckmann M, Schwab ME, Stuermer CAO (1991) Growth of regenerating goldfish axons is inhibited by rat oligodendrocytes and CNS myelin but not by goldfish optic nerve tract oligodendrocyte-like cells and fish CNS myelin. J Neurosci 11:626-640.

Bastmeyer M, Bähr M, Stuermer CAO (1993) Fish optic nerve oligodendrocytes support axonal regeneration of fish and mammalian retinal ganglion cells. Glia 8:1-12.

Caroni P, Schwab ME (1988a) Two membrane protein fractions from rat central myelin with inhibitory properties for neurite growth and fibroblast spreading. J Cell Biol 106:1281-1288.

Caroni P, Schwab ME (1988b) Antibody against myelin-associated inhibitor of neurite growth neutralizes nonpermissive substrate properties of CNS white matter. Neuron 1:85-96.

Fawcett JW, Rokos J, Bakst I (1989) Oligodendrocytes repel axons and cause axonal growth cone collapse. J Cell Sci 92:93-100.

Fawcett JW, Fersht N, Housden L, Schachner M, Pesheva P (1992) Axonal growth on astrocytes is not inhibited by oligodendrocytes. J Cell Sci 103:571-579. 
Grinspan J, Wrabetz L, Kamholz J (1993) Oligodendrocyte maturation and myelin gene expression in PDGF-treated cultures from rat cerebral white matter. J Neurocytol 22:322-333.

Hart IK, Richardson WD, Bolsover SR, Raff MC (1989a) PDGF and intracellular signaling in the timing of oligodendrocyte differentiation. $\mathrm{J}$ Cell Biol 109:3411-3417.

Hart IK, Richardson WD, Heldin CH, Westermark B, Raff MC (1989b) PDGF receptors on cells of the oligodendrocyte-type-2 astrocyte (O2A) cell lineage. Development 105:595-603.

Heldin CH, Backstrom G, Ostman A, Hammacher A, Ronnstrand L, Rubin K, Nister M, Westermark B (1988) Binding of different dimeric forms of PDGF to human fibroblasts: evidence for two separate receptor types. EMBO J 7:1387-1393.

Johnson PW, Abramow-Newerly W, Seilheimer B, Sadoul R, Tropak MB, Arquint M, Dunn RJ, Schachner M, Roder JC (1989) Recombinant myelin-associated glycoprotein confers neural adhesion and neurite outgrowth function. Neuron 3:377-385.

Lang DM, Rubin BP, Schwab ME, Stuermer CAO (1995) CNS myelin and oligodendrocytes of the Xenopus spinal cord-but not optic nerve-are nonpermissive for axon growth. J Neurosci 15:99-109.

McCarthy KD, De Vellis J (1980) Preparation of separate astrocyte and oligodendrocyte cultures from rat cerebral tissue. $J$ Cell Biol 85:890-902.

McKerracher L, David S, Jackson DL, Kottis V, Dunn RJ, Braun PE (1994) Identification of myelin-associated glycoprotein as a major myelin-derived inhibitor of neurite growth. Neuron 13:805-811.

McKinnon RD, Matsui T, Dubois-Dalcq M, Aaronson SA (1990) FGF modulates the PDGF-driven pathway of oligodendrocyte development. Neuron 5:603-614.

Mukhopadhyay G, Doherty P, Walsh FS, Crocker PR, Filbin MT (1994) A novel role for myelin-associated glycoprotein as an inhibitor of axonal regeneration. Neuron 13:757-767.

Noble M, Murray K, Stroobant P, Waterfield MD, Riddle P (1988) Platelet-derived growth factor promotes division and motility and inhibits premature differentiation of the oligodendrocyte/type-2 astrocyte progenitor cell. Nature 333:560-565.
Nowakowski RS, Lewin B, Miller MW (1989) Bromodeoxyuridine immunohistochemical determination of the length of the cell cycle and the DNA-synthetic phase for an anatomically defined population. J Neurocytol 18:311-318.

Pringle N, Collarini EJ, Mosle MJ, Heldin CH, Westermark B, Richardson WD (1989) PDGF A chain homodimers drive proliferation of bipotential (O-2A) glial progenitor cells in the developing rat optic nerve. EMBO J 8:1049-1056.

Raff M, Miller RH, Noble M (1983) A glial progenitor cell that develops in vitro into an astrocyte or an oligodendrocyte depending on culture medium. Nature 303:390-396.

Raff MC, Abney E, Fok-Seang J (1985) Reconstitution of a developmental clock in vitro: a critical role for astrocytes in the timing of oligodendrocyte differentiation. Cell 42:61-69.

Ranscht B, Clapshaw PA, Price J, Noble M, Seifert W (1982) Development of oligodendrocytes and Schwann cells studied with a monoclonal antibody against galactocerebroside. Proc Natl Acad Sci USA 7:2709-2713.

Richardson WD, Pringle N, Mosley MJ, Westermark B, Dubois-Dalcq M (1988) A role for platelet-derived growth factor in normal gliogenesis in the central nervous system. Cell 53:309-319.

Rozengurt E (1986) Early signals in the mitogenic response. Science 234:161-166.

Schnell L, Schneider R, Kolbeck R, Barde Y-A, Schwab ME (1994) Neurotrophin-3 enhances sprouting of corticospinal tract during development and after adult spinal cord lesion. Nature 367:170-173.

Schwab ME, Caroni P (1988) Oligodendrocytes and CNS myelin are nonpermissive substrates for neurite growth and fibroblast spreading in vitro. J Neurosci 8:2381-2393.

Schwab ME, Kapfhammer J, Bandtlow CE (1993) Inhibitors of neurite growth. Annu Rev Neurosci 16:565-595.

Sommer I, Schachner M (1981) Monoclonal antibodies (O1 to O4) to oligodendrocyte cell surfaces. An immunocytological study in the central nervous system. Dev Biol 83:311-327.

Taylor J, Pesheva P, Schachner M (1993) Influence of janusin and tenascin on growth cone behavior in vitro. J Neurosci Res 35:347-362. 